\title{
Predictors for the Transition from Ductal Carcinoma In Situ to Invasive Breast Cancer in Korean Patients
}

\author{
Eun Young Kim, Kee Hoon Hyun, Yong Lai Park, Chan Heun Park, Sung-Im Do ${ }^{1}$ \\ Departments of Surgery and ${ }^{1}$ Pathology, Kangbuk Samsung Hospital, Sungkyunkwan University School of Medicine, Seoul, Korea
}

Purpose: To identify predictive factors of upstaging from diagnosed ductal carcinoma in situ (DCIS) to invasive cancer after surgical excision. Methods: One hundred seventy-four patients diagnosed with DCIS based on biopsies between January 2009 and December 2014 were evaluated. Patients' clinicopathological variables were assessed to identify predictive factors of invasive carcinoma from final pathology. Results: One hundred seventy-four cases of DCIS were included. Of these, 42 were upstaged to invasive carcinoma on the final excision. Preoperative features such as age 40 years or younger at diagnosis, presence of a palpable mass, ultrasonography (USG)-guided core needle biopsy, tumor size $\geq 20 \mathrm{~mm}$ on USG, high grade DCIS, cribriform DCIS, comedo necrosis, presence of intraluminal calcification, estrogen receptor negativity, progesterone receptor negativity and triple-negative subtype were significantly associated with the risk of invasive carcinoma. Multivariate analysis showed that a tumor size $\geq 20 \mathrm{~mm}$ on USG and triple negative subtype were independently associated with upstaging. Conclusion: Tumor size $\geq 20 \mathrm{~mm}$ on USG and triple-negative subtype were independently associated with the upstaging of DCIS to invasive cancer.

Key Words: Breast neoplasms, Noninfiltrating intraductal carcinoma

\section{INTRODUCTION}

Previous reports indicate that $8 \%$ to $44 \%$ of patients initially diagnosed with ductal carcinoma in situ (DCIS) actually have invasive carcinoma [1-11]. Such uncertainty may cause confusion in treatment plans for DCIS, especially with regard to axillary staging. For DCIS lesions that were found to have invasive components at surgical excision, a sentinel lymph node biopsy (SLNB) may be performed as an additional second surgical procedure. SLNB after a lumpectomy or mastectomy has the associated risks of being an additional surgery, and it is cost-ineffective and difficult to perform lymphatic mapping [12,13].

Predictive risk factors of upstaging from DCIS to invasive cancer after surgical excision have been reported, and tumor size is the most widely accepted factor $[8,14]$. In order to avoid a second operation on the axilla if an invasive cancer is found, the American Society of Clinical Oncology recommends SLNB for DCIS in two cases: (1) mastectomy and (2) large ( $>5 \mathrm{~cm}$ ) tumor size with breast-conserving surgery [15]. German guidelines recommend SLNBs in patients with tumors

Correspondence: Chan Heun Park

Department of Surgery, Kangbuk Samsung Hospital, Sungkyunkwan University School of Medicine, 29 Saemunan-ro, Jongno-gu, Seoul 03181, Korea Tel: +82-2-2001-1730, Fax: +82-2-2001-1942, E-mail: chanheun1@gmail.com Received:Dec 9, 2015 Revised: Jan 15, 2016 Accepted:Mar 3, 2016 larger than $5 \mathrm{~cm}$ or in patients with high grade lesions larger than 2.5 $\mathrm{cm}[16]$.

Several diagnostic modalities are useful for assessing the size of DCIS preoperatively. A recent study has reported that tumor sizes measured with ultrasonography (USG) are associated with upstaging from DCIS to invasive lesions [7]. The size of the DCIS can be measured with mammography (MMG) for the preoperative diagnosis of coexisting invasive diseases. Because annual MMG screening is commonly performed, DCIS is often found as calcifications on MMG [8]. These cases occasionally cannot be detected by USG, in which case they will undergo stereo-guided needle biopsy. Although magnetic resonance imaging (MRI) can be used to detect angiogenic lesions of DCIS, it is also associated with a high rate of false positive results [17-19].

The purpose of the present study was to examine predictive factors including different diagnostic modality tools for upstaging from DCIS to invasive cancer.

\section{METHODS}

All studies were conducted with prior approval from the Institutional Review Board of Kangbuk Samsung Hospital (Approval No. 2013-01-251). Upstaging was defined as the identification of invasive 
or microinvasive foci after surgical excision. Microinvasive carcinoma was defined as an invasive portion $\leq 1 \mathrm{~mm}$. Each patient's demographic, clinical, pathological, and radiological variables were evaluated to identify possible predictors of invasive carcinoma on final pathologic examination. Clinical factors included age, location of tumor, menstrual status at diagnosis, and presence of a palpable mass.

\section{Ultrasonography}

USG was performed using an IU22 (Philips Medical Systems, Bothell, USA) or an Aixplorer (SuperSonic Imagine, Aix-en-Provence, France) equipped with a $12-5 \mathrm{MHz}$ linear-array transducer. The longest tumor diameter on the longitudinal USG view was measured.

\section{Mammography}

MMG was performed in two standard imaging planes - the mediolateral oblique and craniocaudal planes - using either a Senographe DS or Senographe Essential (GE Healthcare, Milwaukee, USA) unit. Magnification and spot compression views were obtained for all calcified lesions. Two breast radiologists, each with at least 10 years of experience, evaluated the subjects' lesions according to the Breast Imaging Reporting and Data System (BI-RADS) classification [11].

\section{Magnetic resonance imaging}

All breast MRI examinations were performed using the Intera 1.5T system (Philips Healthcare, Best, The Netherlands) and a four-channel bilateral breast surface coil while patients were in the prone position. The largest of these three diameters was considered a measure of the lesion's size. Morphological analysis was reported according to the BI-RADS classification. To avoid a biased reading, all MRI images were retrospectively reviewed by two breast radiologists with at least 10 years of experience each who were blinded to USG, MMG, and clinical information.

\section{${ }^{18}$ F-FDG PET-CT}

All patients fasted for at least 6 hours prior to scanning and presented with a blood glucose level $<150 \mathrm{mg} / \mathrm{dL} .{ }^{18} \mathrm{~F} 2$-fluoro-2-deoxy-Dglucose integrated positron emission and computed tomography $\left({ }^{18} \mathrm{~F}\right.$ FDG PET-CT) was acquired using two scanners (Discovery ST or STE PET/CT; General Electric Medical Systems, Milwaukee, USA) after an intravenous injection of 370 to $555 \mathrm{MBq}(10-15 \mathrm{mCi}){ }^{18} \mathrm{~F}-\mathrm{fluo}-$ rodeocyglucose $\left({ }^{18} \mathrm{~F}\right.$-FDG) 60 minutes before scanning. Positive FDG uptake was defined as when the radioactivity was higher than that of the surrounding normal tissue in the visual analysis. Images were assessed semi-quantitatively by measuring and calculating the maximum standardized uptake values $\left(\mathrm{SUV}_{\max }\right)$ normalized to lean body mass.

\section{Image-guided biopsy techniques}

Three diagnostic methods were employed: (1) 108 of 174 (62\%) underwent the USG-guided method using 14-gauge needle core biopsies (TSK Ace-cut, Surecut, Stericut; Create Medic Co. Ltd., Yokohama, Japan); (2) 20 of 174 (12\%) underwent stereotactic biopsies using 9-gauge needle core biopsies (Mammotome, SCM 23; Ethicon EndoSurgery Inc., Cincinnati, USA); and (3) 46 of 174 (26\%) underwent wire localization open surgical biopsies $(p=0.041)$. Core needle biopsy $(\mathrm{CNB})$ was considered when a mass-forming shape was found using USG. At least five specimens from different areas of the lesion, each specimen having diameter of $1 \mathrm{~cm}$ on its longest side, were obtained using the 14-gauge needle on USG-guided biopsy. All CNBs were performed by three dedicated breast radiologists with clinical experience ranging from 5 to 20 years. The decision to use a stereotactic biopsy depended largely on the presence of the microcalcifications and a nonmass forming shape detected with USG. The number of cores sampled was recorded. All stereotactic biopsies for calcifications were accompanied by a specimen radiograph that demonstrated capture of the target lesion in the specimen. Wire localization open surgical biopsy was considered when microcalcification was not detected in USG.

Pathological features included grade (low, intermediate, high, and not specified), histological type (cribriform, papillary, solid, and not specified), type of necrosis (comedo, noncomedo, and no necrosis), and presence of intraluminal calcification on the CNB.

Following the biopsy of DCIS, all patients underwent surgery in the form of a simple mastectomy or wide excision, with a SLNB. Cases were divided into DCIS (Group 1) and invasive (Group 2) groups based on the histological results from all operated specimens. The invasive group also included cases of microinvasion.

All biopsy materials were immunostained with antibodies to the estrogen receptor (ER; 1/200, SP1 Clone; Lab Vision Corp., Fremont, USA), progesterone receptor (PR; 1/200, PgR636Clone; DAKO, Glostrup, 
Denmark), and human epithelial growth factor receptor 2 (HER2; 1/200; A 0485 Clone; DAKO) and were evaluated under the microscope by two pathologists with 10 years of experience each. All tissues were also immunostained with antibodies to $\mathrm{p} 63$, which is a sensitive and specific myoepithelial marker. We aimed to identify an invasive focus by the lack of myoepithelial cells using immunostaining. An Allred score of $\geq 3$ for ER and PR nuclear immunoreactivity was considered positive. A 3+ membrane score for HER2 staining was considered positive. Fluorescence in situ hybridization (FISH), which tests for HER2 gene amplification, was considered in cases with scores of $2+$. If $>10 \%$ of tumor cells were found in the FISH test, then a membrane score of $2+$ was considered a positive result.

\section{Statistics}

Qualitative variables were compared using Fisher exact test. Normally distributed quantitative variables were compared using Student t-test, while nonnormally distributed quantitative variables were compared using the Mann-Whitney U-test. Univariate and multivariate

Table 1. Patients' clinical, pathological, and radiological characteristics

\begin{tabular}{|c|c|c|c|c|c|c|c|}
\hline Characteristic & $\begin{array}{c}\text { DCIS } \\
(n=132) \\
\text { No. }(\%)\end{array}$ & $\begin{array}{c}\text { Invasive cancer } \\
(n=42) \\
\text { No. }(\%)\end{array}$ & & Characteristic & $\begin{array}{c}\text { DCIS } \\
(n=132) \\
\text { No. }(\%)\end{array}$ & $\begin{array}{l}\text { Invasive cancer } \\
\qquad(n=42) \\
\text { No. }(\%)\end{array}$ & \\
\hline Age $(y r)^{*}$ & $51.611 \pm 9.960$ & $50.112 \pm 11.064$ & 0.437 & M stage & & & \\
\hline$\leq 40$ & $14(10.6)$ & $10(23.8)$ & & 0 & & $42(100)$ & \\
\hline$>40$ & $118(89.4)$ & $32(76.2)$ & & 1 & & 0 & \\
\hline Tumor location & & & 0.990 & USG BI-RADS & & & 0.002 \\
\hline Left & $63(47.7)$ & $20(47.6)$ & & 1 & $2(1.5)$ & $1(2.4)$ & \\
\hline Right & $69(52.3)$ & $22(52.4)$ & & 2 & $4(3.0)$ & $1(2.4)$ & \\
\hline Postmenopausal & & & 0.447 & 3 & $9(6.8)$ & $3(7.1)$ & \\
\hline Yes & $51(38.6)$ & $19(45.2)$ & & 4 & $97(73.6)$ & $20(47.6)$ & \\
\hline No & $81(61.4)$ & $23(54.8)$ & & 5 & $12(9.1)$ & $15(35.7)$ & \\
\hline Presence of palpable mass & & & 0.010 & 6 & $2(1.5)$ & 0 & \\
\hline Yes & $43(32.6)$ & $23(54.8)$ & & Unknown & $6(4.5)$ & $2(4.8)$ & \\
\hline No & $89(67.4)$ & $19(45.2)$ & & MMG BI-RADS & & & 0.080 \\
\hline Multiple lesions on USG & & & 0.775 & 0 & $12(9.1)$ & $5(11.9)$ & \\
\hline Yes & 14 (10.6) & $7(16.7)$ & & 1 & $7(5.3)$ & $3(7.1)$ & \\
\hline No & $118(89.4)$ & $35(83.3)$ & & 2 & $3(2.3)$ & 0 & \\
\hline Contralateral lesions on MRI & & & 0.631 & 3 & $1(0.8)$ & $1(2.4)$ & \\
\hline Yes & $25(18.9)$ & $5(11.9)$ & & 4 & $70(53.0)$ & $16(38.1)$ & \\
\hline No & $107(81.1)$ & $37(88.1)$ & & 5 & $10(7.6)$ & $10(23.8)$ & \\
\hline Size of target lesion on USG & $14.343 \pm 11.072$ & $24.431 \pm 15.120$ & $<0.001$ & 6 & $7(5.3)$ & $1(2.4)$ & \\
\hline$(\mathrm{mm})^{*}$ & & & & Unknown & $22(16.6)$ & $6(14.3)$ & \\
\hline Size of target lesion on MMG & $20.641 \pm 17.352$ & $28.202 \pm 22.204$ & 0.074 & MRI BI-RADS & & & 0.432 \\
\hline$(\mathrm{mm})^{*}$ & & & & 4 & $2(1.5)$ & $2(4.8)$ & \\
\hline Size of target lesion on MRI & $23.361 \pm 17.234$ & $33.794 \pm 17.590$ & 0.004 & 5 & $5(3.8)$ & $1(2.4)$ & \\
\hline$(\mathrm{mm})^{*}$ & & & & 6 & $103(78.0)$ & $31(73.8)$ & \\
\hline Tstage & & & & Unknown & $22(16.7)$ & $8(19.0)$ & \\
\hline 1 & & $42(100)$ & & SUV $_{\max }$ of ${ }^{18} \mathrm{~F}$-FDG PET-CT ${ }^{*}$ & $2.301 \pm 1.040$ & $2.772 \pm 1.331$ & 0.094 \\
\hline 2 & & 0 & & Type of final operation & & & 0.001 \\
\hline 3 & & 0 & & Breast-conserving surgery & $70(53.0)$ & $9(21.4)$ & \\
\hline 4 & & 0 & & Mastectomy & $62(47.0)$ & 33 (78.6) & \\
\hline \multicolumn{8}{|l|}{$\mathrm{N}$ stage } \\
\hline 0 & & $38(90.4)$ & & & & & \\
\hline 1 & & $4(9.6)$ & & & & & \\
\hline 2 & & 0 & & & & & \\
\hline 3 & & 0 & & & & & \\
\hline
\end{tabular}

$\mathrm{DCIS}=$ ductal carcinoma in situ; USG = ultrasonography; $\mathrm{MRI}=$ magnetic resonance imaging; $\mathrm{MMG}=$ mammography; $\mathrm{BI}-\mathrm{RADS}=\mathrm{Breast}$ Imaging Reporting and Data System; $\mathrm{SUV}_{\max }=$ maximum standardized uptake values; ${ }^{18} \mathrm{~F}$-FDG PET-CT $={ }^{18} \mathrm{~F}$ 2-fluoro-2-deoxy-D-glucose integrated positron emission and computed tomography.

${ }^{*}$ Mean \pm SE. 
analyses were performed to assess the predictive value of the variables. All statistical analyses were performed using IBM SPSS Statistics software 21.0 (IBM Corp., Armonk, USA), with statistical significance set at $p<0.05$.

\section{RESULTS}

One hundred seventy-four cases of DCIS were included in the study. Of these, 42 (24\%) were upstaged to invasive carcinoma on the final excision. The patients were divided into two groups according to

Table 2. Methods and results of biopsy

\begin{tabular}{|c|c|c|c|}
\hline Characteristic & $\begin{array}{c}\text { DCIS } \\
(n=132) \\
\text { No. }(\%)\end{array}$ & $\begin{array}{l}\text { Invasive cancer } \\
(\mathrm{n}=42) \\
\text { No. }(\%)\end{array}$ & $p$-value \\
\hline No. of biopsy* & $7.680 \pm 6.741$ & $6.762 \pm 6.434$ & 0.495 \\
\hline Type of biopsy & & & 0.002 \\
\hline USG-guided CNB & $73(55.3)$ & $35(83.3)$ & \\
\hline Stereotactic biopsy & $17(12.9)$ & $3(7.1)$ & \\
\hline $\begin{array}{l}\text { Wire localization open } \\
\text { surgical biopsy }\end{array}$ & $42(31.8)$ & $4(9.6)$ & \\
\hline Histologic grade & & & 0.077 \\
\hline Low & $36(27.3)$ & $5(11.9)$ & \\
\hline Intermediate & $53(40.2)$ & $15(35.7)$ & \\
\hline High & $39(29.5)$ & $18(42.8)$ & \\
\hline Unknown & $4(3.0)$ & $4(9.6)$ & \\
\hline DCIS subtype & & & 0.238 \\
\hline Cribriform & $71(53.8)$ & $13(30.9)$ & \\
\hline Papillary & $10(7.6)$ & $4(9.6)$ & \\
\hline Solid & $25(18.9)$ & $11(26.2)$ & \\
\hline Unknown & $26(19.7)$ & $14(33.3)$ & \\
\hline $\begin{array}{l}\text { Presence of intraluminal } \\
\text { calcification }\end{array}$ & & & 0.008 \\
\hline Yes & $76(57.6)$ & $15(35.7)$ & \\
\hline No & $56(42.4)$ & $27(64.3)$ & \\
\hline Presence of necrosis & & & 0.033 \\
\hline Comedo necrosis & $49(37.1)$ & $24(57.1)$ & \\
\hline Noncomedo necrosis & $29(21.9)$ & $8(19.0)$ & \\
\hline No necrosis & $54(41.0)$ & $10(23.9)$ & \\
\hline \multicolumn{4}{|l|}{ Hormonal status } \\
\hline ER-positive & $94(71.2)$ & $18(42.9)$ & 0.010 \\
\hline PR-positive & $85(64.4)$ & $16(38.1)$ & 0.001 \\
\hline HER2 overexpression & $57(43.2)$ & $23(54.8)$ & 0.745 \\
\hline Triple negative & $3(2.3)$ & $5(11.9)$ & 0.024 \\
\hline Ki-67 expression* & $8.533 \pm 9.694$ & $11.320 \pm 7.731$ & 0.115 \\
\hline $\begin{array}{l}\text { Mean pathologic DCIS size } \\
(\mathrm{mm})^{*}\end{array}$ & $19.991 \pm 17.310$ & $30.334 \pm 24.732$ & 0.003 \\
\hline
\end{tabular}

DCIS = ductal carcinoma in situ; USG = ultrasonography; $C N B=$ core needle biopsy; $E R=$ estrogen receptor; $\mathrm{PR}=$ progesterone receptor; $\mathrm{HER} 2$ = human epithelial growth factor receptor 2 .

*Mean \pm SE. presence of an invasive component on final pathologic examination. The clinical, pathological, and radiological characteristics of the two groups are shown (Table 1). Eighty-nine percent (118/132) of the DCIS group and 76\% (32/42) of the invasive cancer group were older than 40 years old. The mean diameters of the two groups measured using USG, MMG, and MRI are shown in Table 1. The invasive cancer group had a larger target size measured using USG and MRI than the DCIS group $(p<0.05)$. In total, $52 \%(65 / 125)$ of the DCIS group underwent breast-conserving surgery, while 75\% (30/39) of the invasive cancer group underwent a mastectomy.

In the DCIS and invasive cancer groups, 55\% and 83\%, respectively, underwent USG-guided CNB (Table 2). Intraluminal calcification was observed in $56 \%$ of the DCIS group and $32 \%$ of the invasive cancer group, and 37\% of DCIS group and 60\% of invasive cancer group had comedo necrosis. Thirty-nine percent of the DCIS group was ERpositive, and $29 \%$ of the invasive cancer group had HER 2 overexpression.

Univariate analysis showed that an age 40 years or younger at diagnosis, presence of a palpable mass, USG guided CNB, tumor size $\geq 20$ mm on USG, high-grade DCIS, cribriform DCIS, comedo necrosis, presence of intraluminal calcification, ER negativity, PR negativity, and triple negative subtype were significant risk factors for invasive disease $(p<0.05$ for each variable) (Table 3$)$. Multivariate analysis showed that tumor size $\geq 20 \mathrm{~mm}$ on USG and triple negative subtype were independently associated with upstaging $(p<0.05$ for each variable) (Table 3).

\section{DISCUSSION}

Theoretically, pure DCIS is a localized disease confined within the basement membrane of the breast duct without the risk of invasion into lymph nodes or vessels. Therefore, axillary nodal staging and surgical management are unnecessary for pure DCIS without invasive components. However, histological underestimation of invasive carcinoma diagnosed as DCIS often occurs due to several reasons. First, the increased use of percutaneous CNB and likelihood of missed invasive breast cancer by $\mathrm{CNB}$ have increased [12]. CNB is a highly reliable tool in diagnosing breast cancers, and it is also cost-effective, since it reduces the number of surgical procedures required for breast cancer patients [20]. Despite these advantages, CNB may lead to histo- 
Table 3. Analysis of predictors for invasive breast cancer from DCIS in image guided biopsy using univariate and multivariate logistic regression models

\begin{tabular}{|c|c|c|c|c|}
\hline & \multicolumn{2}{|c|}{ Univariate logistic regression } & \multicolumn{2}{|c|}{ Multivariate logistic regression } \\
\hline & OR $(95 \% \mathrm{Cl})$ & $p$-value & OR $(95 \% \mathrm{Cl})$ & $p$-value \\
\hline Age $\leq 40 \mathrm{yr}$ & $2.633(1.070-6.482)$ & 0.035 & $3.850(0.889-6.671)$ & 0.071 \\
\hline Postmenopausal & $1.312(0.650-2.645)$ & 0.448 & & \\
\hline Presence of palpable mass & $2.505(1.233-5.087)$ & 0.011 & $1.564(0.514-4.752)$ & 0.430 \\
\hline USG-guided CNB & $4.041(1.674-9.753)$ & 0.002 & & \\
\hline Stereotactic biopsy & $0.362(0.079-1.646)$ & 0.189 & & \\
\hline Wire localization open surgical biopsy & $0.225(0.755-0.673)$ & 0.080 & & \\
\hline Size of target lesion on USG $\geq 20 \mathrm{~mm}$ & $4.891(2.159-11.082)$ & 0.001 & $8.127(2.415-27.353)$ & 0.001 \\
\hline High ultrasonography BI-RADS $(4,5,6)$ & $0.945(0.320-2.788)$ & 0.920 & & \\
\hline High MMG BI-RADS $(4,5,6)$ & $0.793(0.327-1.918)$ & 0.607 & & \\
\hline SUV $_{\max } \geq 2$ & $2.571(0.986-6.701)$ & 0.053 & & \\
\hline High grade DCIS on biopsy & $2.053(0.980-4.303)$ & 0.047 & $0.622(0.161-2.401)$ & 0.491 \\
\hline \multicolumn{5}{|l|}{ Type of DCIS } \\
\hline Cribriform & $0.427(0.183-0.995)$ & 0.049 & $0.360(0.117-1.100)$ & 0.073 \\
\hline Papillary & $1.600(0.461-5.544)$ & 0.459 & & \\
\hline Solid & $2.096(0.868-5.059)$ & 0.100 & & \\
\hline \multicolumn{5}{|l|}{ Presence of necrosis } \\
\hline Noncomedo & $1.758(0.596-5.182)$ & 0.306 & & \\
\hline Comedo & $3.122(1.280-7.611)$ & 0.012 & $4.379(0.941-20.383)$ & 0.060 \\
\hline Presence of intraluminal calcification & $2.707(1.281-5.718)$ & 0.009 & $2.116(0.668-6.700)$ & 0.202 \\
\hline No. of core biopsy $\leq 5$ & $1.785(0.685-4.649)$ & 0.235 & & \\
\hline ER-negativity & $3.580(1.736-7.385)$ & 0.001 & $1.562(0.222-10.960)$ & 0.653 \\
\hline PR-negativity & $3.319(1.525-6.457)$ & 0.002 & $1.344(0.177-10.246)$ & 0.775 \\
\hline HER2 overexpression & $1.125(0.551-2.297)$ & 0.745 & & \\
\hline Triple negative & $5.540(1.264-24.28)$ & 0.023 & $17.157(1.243-23.677)$ & 0.034 \\
\hline Multiple lesions on MRI & $0.611(0.271-1.374)$ & 0.234 & & \\
\hline
\end{tabular}

$\mathrm{DCIS}=$ ductal carcinoma in situ; $\mathrm{OR}=$ odds ratio; $\mathrm{Cl}=$ confidence interval; USG = ultrasonography; $\mathrm{CNB}=$ core needle biopsy; BI-RADS = Breast Imaging Reporting and Data System; $M M G=$ mammography; $S_{U V} V_{\max }=$ maximum standardized uptake values; $E R=$ estrogen receptor; $P R=$ progesterone receptor; $\mathrm{HER} 2$ = human epithelial growth factor receptor $2 ; \mathrm{MRI}=$ magnetic resonance imaging.

logical underestimation, with invasive carcinoma diagnosed as DCIS due to insufficient sampling. Such patients may then be required to undergo an additional operation for axillary staging, and the accuracy of this second operation may be compromised by lymphatic disruption or inflammatory changes resulting from the initial operation for DCIS $[13,16,21]$. Therefore, the purpose of this study was to identify factors leading to upstaging invasive cancer from DCIS on CNB.

Forty-two patients (24\%) were upstaged to invasive carcinoma on final excision. The higher underestimation rate in our study, compared with other previous studies [14,15], may be attributed in part to the small sample size and an insufficient number of biopsies (Table 2).

We also analyzed whether pathological features were associated with the upstaging of breast carcinoma. High histological grade DCIS has been known as another risk factor. According to previous reports, high-grade, poorly differentiated DCIS has been associated with a 3 -fold higher risk of tumor invasion $[6,8,22]$. We also found that high histological grade was associated with an estimated two-fold risk of invasiveness on univariate analysis (odds ratio [OR], 2.053; $p=0.047$ ) (Table 3).

Comedo necrosis also has been reported as a risk factor of upstaging but its significance has been controversial [17]. In our study, it was a significant risk factor for tumor upstaging $(\mathrm{OR}, 3.122 ; p=0.012)(\mathrm{Ta}-$ ble 3). Renshaw [18] reported that patients with comedo DCIS with a cribriform/papillary pattern or a tumor involving more than $4 \mathrm{~mm}$ with lobular extension in breast core needle specimens are at increased risk for invasion at excision.

Analyses of immunohistochemical features as predictors of invasive disease have been published [19]. According to these studies, hormone receptor positivity has been related to low-grade DCIS. HER2 overexpression was known to be superior to lesion size or histological grade in predicting concurrent invasive disease in a recent case series [23]. We found that the ORs were 3.580 for ER negativity, 3.319 for PR 
negativity, 1.125 for HER2/neu overexpression, and 5.540 for triplenegative DCIS (Table 3). Lee et al. [21] reported lack of hormone receptor expression of DCIS as predictive factor for upstaging, and these results are consistent with ours.

Since recent research about the expression of hormone receptors in DCIS has mainly focused on the risk of local recurrence following surgery [22], results about hormone receptor status are rare. There is still a controversy about whether immunohistochemical features predict recurrence or invasion independent of other factors in DCIS, and further studies are warranted.

Several studies have found that a large preoperative tumor size is the most generally accepted risk factor [7]. The size of DCIS is not only a predictor of tumor upstaging, but it is also important in determining the extent of surgical treatment.

Tumor size measured using USG (OR, 4.891; $p=0.001$ ) (Table 3) was found to be a significant predictor of upstaging. Since breast tissue density is generally higher in Asian than in Caucasian women, diagnostic USG is frequently performed in our institution together with screening MMG [24]. A recent study showed results consistent with ours [10]. Therefore, the simultaneous measurement of a DCIS lesion with USG-guided CNB may help to determine the optimal surgical treatment plan.

An age of 40 years or younger at diagnosis was significantly associated with the presence of invasive disease at final pathology $(\mathrm{OR}$, $2.633 ; p=0.035)$ in univariate analysis. Other studies found that younger patients with DCIS generally had unfavorable prognostic factors and were at a greater risk of adverse events [25]. The finding that younger age was a predictor of invasive cancer is explained by the fact that younger age is also associated with other adverse clinical factors for invasive disease, including higher tumor grade, presence of necrosis, more extensive disease, and clinical findings [26].

In the present study, cribriform subtype of DCIS was negatively correlated with upstaging (Table 3). Several authors have reported relationships between different DCIS subtypes, the comedo and cribriform/papillary type, and subsequent upstaging to invasive disease [18]. However, there is no consensus regarding the association between pathological subtypes of DCIS and upstaging to invasive disease.

There was a difference in the rates of upstaging based on the $\mathrm{CNB}$ technique (Table 3). The USG-guided method using a 14-gauge nee- dle was found to be a significant predictor of upstaging. The diagnostic biopsy needle size dictates how much tumor tissue can be sampled [27,28]. Another study found that the use of a smaller needle (i.e., the gun method compared to the vacuum method) was associated with a higher risk of upstaging [29].

This study has several limitations. First, it was performed at a single institution and was retrospective in design. Several patients underwent film MMG before 2010, which has lower sensitivity and specificity for cancer detection.

Age 40 years or younger at diagnosis, presence of a palpable mass, USG guided CNB, high grade DCIS, cribriform DCIS, comedo necrosis, presence of intraluminal calcification, ER negativity, and PR negativity were risk factors. Tumor size $\geq 20 \mathrm{~mm}$ on USG and triple negative subtype were independently associated with the upstaging of DCIS to invasive cancer. In particular, the size measured with USG was associated with upstaging, whereas size measurement by MG, $\mathrm{MRI}$, and ${ }^{18} \mathrm{~F}$-FDG PET-CT was not. Further studies of larger populations are needed to determine the predictive value of immunohistochemical features and their relationship with tumor upstaging.

\section{CONFLICT OF INTEREST}

The authors declare that they have no competing of interests.

\section{REFERENCES}

\section{Darling ML, Smith DN, Lester SC, Kaelin C, Selland DL, Denison}

$\mathrm{CM}$, et al. Atypical ductal hyperplasia and ductal carcinoma in situ as revealed by large-core needle breast biopsy: results of surgical excision. AJR Am J Roentgenol 2000;175:1341-6.

2. Wahedna Y, Evans AJ, Pinder SE, Ellis IO, Blamey RW, Geraghty JG. Mammographic size of ductal carcinoma in situ does not predict the presence of an invasive focus. Eur J Cancer 2001;37:459-62.

3. Jackman RJ, Burbank F, Parker SH, Evans WP 3rd, Lechner MC, Richardson TR, et al. Stereotactic breast biopsy of nonpalpable lesions: determinants of ductal carcinoma in situ underestimation rates. Radiology 2001;218:497-502.

4. King TA, Farr GH Jr, Cederbom GJ, Smetherman DH, Bolton JS, Stolier AJ, et al. A mass on breast imaging predicts coexisting invasive carcinoma in patients with a core biopsy diagnosis of ductal 
carcinoma in situ. Am Surg 2001;67:907-12.

5. Cox D, Bradley S, England D. The significance of mammotome core biopsy specimens without radiographically identifiable microcalcification and their influence on surgical management: a retrospective review with histological correlation. Breast 2006;15:210-8.

6. Hoorntje LE, Schipper ME, Peeters PH, Bellot F, Storm RK, Borel Rinkes IH. The finding of invasive cancer after a preoperative diagnosis of ductal carcinoma-in-situ: causes of ductal carcinoma-in-situ underestimates with stereotactic 14-gauge needle biopsy. Ann Surg Oncol 2003;10:748-53.

7. Yen TW, Hunt KK, Ross MI, Mirza NQ, Babiera GV, Meric-Bernstam F, et al. Predictors of invasive breast cancer in patients with an initial diagnosis of ductal carcinoma in situ: a guide to selective use of sentinel lymph node biopsy in management of ductal carcinoma in situ. J Am Coll Surg 2005;200:516-26.

8. Wilkie C, White L, Dupont E, Cantor A, Cox CE. An update of sentinel lymph node mapping in patients with ductal carcinoma in situ. Am J Surg 2005;190:563-6.

9. Han JS, Molberg KH, Sarode V. Predictors of invasion and axillary lymph node metastasis in patients with a core biopsy diagnosis of ductal carcinoma in situ: an analysis of 255 cases. Breast J 2011; $17: 223-9$

10. Houssami N, Ambrogetti D, Marinovich ML, Bianchi S, Macaskill P, Vezzosi V, et al. Accuracy of a preoperative model for predicting invasive breast cancer in women with ductal carcinoma-in-situ on vacuum-assisted core needle biopsy. Ann Surg Oncol 2011;18: 1364-71.

11. Miyake T, Shimazu K, Ohashi H, Taguchi T, Ueda S, Nakayama T, et al Indication for sentinel lymph node biopsy for breast cancer when core biopsy shows ductal carcinoma in situ. Am J Surg 2011;202:5965.

12. Tan JC, McCready DR, Easson AM, Leong WL. Role of sentinel lymph node biopsy in ductal carcinoma-in-situ treated by mastectomy. Ann Surg Oncol 2007;14:638-45.

13. Feldman SM, Krag DN, McNally RK, Moor BB, Weaver DL, Klein P. Limitation in gamma probe localization of the sentinel node in breast cancer patients with large excisional biopsy. J Am Coll Surg 1999;188:248-54.

14. Dillon MF, McDermott EW, Quinn CM, O’Doherty A, O’Higgins

$\mathrm{N}$, Hill AD. Predictors of invasive disease in breast cancer when core biopsy demonstrates DCIS only. J Surg Oncol 2006;93:559-63.

15. Lyman GH, Giuliano AE, Somerfield MR, Benson AB 3rd, Bodurka DC, Burstein HJ, et al. American Society of Clinical Oncology guideline recommendations for sentinel lymph node biopsy in early-stage breast cancer. J Clin Oncol 2005;23:7703-20.

16. van Deurzen CH, Hobbelink MG, van Hillegersberg R, van Diest PJ. Is there an indication for sentinel node biopsy in patients with ductal carcinoma in situ of the breast? A review. Eur J Cancer 2007; 43:993-1001.

17. Schouten van der Velden AP, Boetes C, Bult P, Wobbes T. The value of magnetic resonance imaging in diagnosis and size assessment of in situ and small invasive breast carcinoma. Am J Surg 2006;192: $172-8$.

18. Renshaw AA. Predicting invasion in the excision specimen from breast core needle biopsy specimens with only ductal carcinoma in situ. Arch Pathol Lab Med 2002;126:39-41.

19. Sakorafas GH, Farley DR, Peros G. Recent advances and current controversies in the management of DCIS of the breast. Cancer Treat Rev 2008;34:483-97.

20. Smith DN, Christian R, Meyer JE. Large-core needle biopsy of nonpalpable breast cancers: the impact on subsequent surgical excisions. Arch Surg 1997;132:256-9.

21. Lee SK, Yang JH, Woo SY, Lee JE, Nam SJ. Nomogram for predicting invasion in patients with a preoperative diagnosis of ductal carcinoma in situ of the breast. Br J Surg 2013;100:1756-63.

22. Bagnall MJ, Evans AJ, Wilson AR, Pinder SE, Denley H, Geraghty JG, et al. Predicting invasion in mammographically detected microcalcification. Clin Radiol 2001;56:828-32.

23. Roses RE, Paulson EC, Sharma A, Schueller JE, Nisenbaum H, Weinstein S, et al. HER-2/neu overexpression as a predictor for the transition from in situ to invasive breast cancer. Cancer Epidemiol Biomarkers Prev 2009;18:1386-9.

24. Crystal P, Strano SD, Shcharynski S, Koretz MJ. Using sonography to screen women with mammographically dense breasts. AJR Am J Roentgenol 2003;181:177-82.

25. Trentin C, Dominelli V, Maisonneuve P, Menna S, Bazolli B, Luini A, et al. Predictors of invasive breast cancer and lymph node involvement in ductal carcinoma in situ initially diagnosed by vacuum-assisted breast biopsy: experience of 733 cases. Breast 2012;21:635-40. 26. Burstein HJ, Polyak K, Wong JS, Lester SC, Kaelin CM. Ductal car- 
cinoma in situ of the breast. N Engl J Med 2004;350:1430-41.

27. Tse GM, Tan PH. Diagnosing breast lesions by fine needle aspiration cytology or core biopsy: which is better? Breast Cancer Res Treat 2010;123:1-8.

28. Green S, Khalkhali I, Azizollahi E, Venegas R, Jalil Y, Dauphine C. Excisional biopsy of borderline lesions after large bore vacuum-as- sisted core needle biopsy: is it necessary? Am Surg 2011;77:1358-60.

29. Kim J, Han W, Lee JW, You JM, Shin HC, Ahn SK, et al. Factors associated with upstaging from ductal carcinoma in situ following core needle biopsy to invasive cancer in subsequent surgical excision. Breast 2012;21:641-5. 